\title{
Healthcare Evidence-Based Management: Towards Overcoming its Barriers in Uganda's Local Government Healthcare System
}

\author{
Article by Stanley T. Asaku ${ }^{1}$, Gerald K. Karyeija ${ }^{2}$, Fred Alinda ${ }^{2}$, Kennedy O. Ojwang, \\ Martine D. Andua ${ }^{3}$ and Patrick Y. Anguzu ${ }^{3}$ \\ ${ }^{1}$ School of Public Health, Texila American University, Guyana, South America \\ ${ }^{2}$ Department of Political and Administrative Sciences, School of Management Sciences, \\ Uganda Management Institute (UMI), Uganda \\ ${ }^{3}$ Directorate of Health Services, Arua District Local Government, West Nile Subregion, \\ Uganda \\ Email: coopdev.tstanley@gmail.com1
}

\begin{abstract}
This article identifies local barriers and potential promoters of healthcare evidence-based management decision-making in Uganda's local government context. It puts to local context feasible measures for increasing research evidence utilization by healthcare decision-makers, as part of the efforts to make research more beneficial to intended users, and ultimate recipients of the services. The findings were a result of a cross- sectional semi-structured questionnaire survey of 225 clustered healthcare authorities ${ }^{l}$ in Arua District Local Government, West Nile Sub-region of Uganda. The survey data were triangulated with nine key informant interviews (KIIs). Analysis reveals existence of multiple barriers at individual, organizational and system's levels of the local government healthcare management. Standing out prominently were barriers related to attitude, perceptions and beliefs of healthcare managers, dissemination, accessibility, communication, participation, engagement, capacity, knowledge, skills, cost, time, staffing, workload, leadership, policy enforcement, and culture. Other barriers related to researchers were their competence, authority and level of mutual trust. Fortunately, most of these barriers are consistent with those reported previously by other studies in developing countries. Through a critical logical analysis, recommended strategies for increasing utilization of research evidence were combined into five broad categories; stakeholders' engagement and participation, contextualized dissemination, capacity building, local leadership and democracy, and knowledge marketing, awareness and visibility. Again, these are not naïve, but important is the manner and details in which they have been contextualized. Hence, this article adds to existing knowledge about multifactorial contextual nature of barriers and promoters of research evidence utilization, and the importance of action research in providing evidence for improving quality of healthcare service delivery.
\end{abstract}

Keywords: barriers, promoters, research evidence, healthcare authorities.

\section{Introduction}

Healthcare management decisions should preferably be based on high quality research evidence, but despite increased public investments in healthcare research that has resulted in the subsequent increase in wealth of credible knowledge available for making evidence-based management decisions (Melnyk et al., 2012), studies continue to report lower than expected levels of research utilization across all levels of healthcare management in both developed and developing countries. The disparity between amount of evidence that is available and its use is called a research-practice (Squires et al., 2011). Research evidence use continues to be a global concern, with considerable difficulty putting it to practice (Amanda et al., 2009).

Pfeffer and Sulton (2006a) assert that though thousands of studies are conducted every year, little appears to be utilized, with only less than 20 per cent of all management decisions take into account scientific evidence whilst the rest are made absolutely on basis of obsolete knowledge gained way back in school, traditions, experience, beliefs or other sources of information. Likewise, Melnyk et al. (2012) decries that research evidence use remains slow and unpredictable, or even worse among

\footnotetext{
${ }^{1}$ Healthcare managers, administrators and political leaderships
} 
managers, whom according to Fraser and Clancy (2007), and Pfeffer and Sulton (2006a) have been much slower, more-so in developing countries where efforts to promote evidence-based management (EBMgt) has faced greater challenges.

The concept of EBMgt is relatively recent, often associated with Stanford professors Jeff Pfeffer and Bob Sutton (2006a \& 2006b), whose book and article in the Harvard Business Review drew considerable attention to the subject. However, the notion has its origin in the broader concept of evidence-based practice, which can be traced back to evidence-based medicine, a set of principles and methods intended to ensure that to the greatest extent possible, healthcare decisions, guidelines, and policies are based on and are consistent with best available research evidence (JEBMH, 2014). The object is to arrive at best decisions and improve quality of healthcare systems upon integrating best scientific evidence, professional expertise, and values as well as preferences of final recipients (Sackett et al., 1996; Straus et al., 2005). Unfortunately, the adoption of scientific evidence still appears far from reality, as the gap between practice and evidence use persists (Rengerink et al., 2013).

There are six basic steps or rather commonly referred to as 6As' for utilizing research evidence (Barends et al., 2014) entails translating practical problem into answerable questions (Asking), systematically searching and retrieving evidences (Accessing), critically appraising these evidences (Appraising), pulling together all relevant evidences (Aggregating), putting to decision action the best evidence (Applying), and finally evaluating the decision outcomes (Assessing).

In short, the philosophy in fronting EBMgt is to move professional decisions away from personal preferences and unsystematic experiences, toward those based on the best scientific evidence (Rousseau, 2006; Pfeffer \& Sulton, 2006b). This however has encountered numerous variable barriers that call for deliberate urgent systematic actions (Rengerink et al., 2013). First, is to identify and document the local barriers and potential promoters, and then disseminate measures towards increased evidence use, putting into consideration the local contexts. This was the purpose of this study, in a local government context of Uganda. We are optimistic that disseminating findings from this study will contribute to promoting research evidence uptake. This is particularly important considering that most studies in literature seem have focused on simply identifying barriers and/or promoters, with less focus on strategies. This is evident in the recent systematic review of barriers to and promoters of research evidence by policy makers (Oliver et al., 2014), in which up to 59.3 per cent of all studies reviewed focused on identifying barriers and/or facilitators, while only 5.5 per cent provided strategies for increasing uptake.

\section{Methodology}

We reviewed existing literature related to barriers and promoters of evidence-based practice, with a focus on use of scientific evidence in healthcare management decisions and practice. We then conducted an empirical semi-structured self-administered questionnaire survey of 225 healthcare authorities, and nine key informant interviews in Arua district local government, West Nile Uganda. The healthcare authorities who participated in this study comprised healthcare line managers (HM), healthcare administrators (ADM), and political leaders, who were known to play key roles in making healthcare management decisions in their work place. Hence, support personnel like drivers, cleaners and interns were not eligible to participate. We pre-tested the questionnaires for internal reliability and validity, and obtained the Cronbach's Coefficient Alpha $(\alpha)$ and mean content validity index (CVI) of 0.91 and 0.96 respectively, which were both higher than the minimum generally accepted (Kline, 2000; Amin 2005).

To achieve this, we divided the study population into 29 clusters, each representing the lower local government administrative sub counties. We determined the average size of the clusters by dividing the total number of healthcare authorities (study population) by total number of clusters i.e. 712/29, which gave 24.5 observation units per cluster. We then divided the study sample size by the average cluster size (i.e. 245/24.5), thereby arriving at 10 as required number of clusters in which to focus the study. We then randomly and blindly selected 10 clusters from the total pool of the 29 clusters, picking one by one each time, until reaching the desired number.

At cluster level, we used the purposive sampling method (Kothari, $2005 \&$ Amin, 2005) to select eligible participants on basis of their roles in making healthcare management decisions. Overall, this sampling method allowed a fair representation of all categories of study population.

Survey responses were rated on basis of the five point Likert scale continuum $(1=$ strongly disagree, $5=$ strongly agree $)$ or the frequency continuum $(1=$ never, $2=$ rarely, $3=$ occasionally, $4=$ frequently, $5=$ almost always). At analysis level, binary responses were generated by combining the 
response categories, allowing the data to be subjected to odd ratios estimation using the binary logistic regression model.

\section{Results}

\section{Demographic statistics}

Table 1 provides a summary of demographic descriptive statistics, in which men constituted 56.4 per cent, youth (44.9\%), and 57.1 per cent had less than 10 years' specialty experience.

Table 1. Descriptive statistics for respondents' background demographic information

\begin{tabular}{|c|c|c|c|}
\hline Variable & Category & Frequency & $\%$ of respondents \\
\hline \multirow{2}{*}{ Gender } & Male & 127 & 56.4 \\
\hline & Female & 98 & 43.6 \\
\hline \multirow{3}{*}{ Age brackets } & $18-34$ years & 101 & 44.9 \\
\hline & $35-52$ years & 102 & 45.4 \\
\hline & Over 52 years & 22 & 9.7 \\
\hline \multirow{8}{*}{ Education level } & Primary leaving certificate & 4 & 1.8 \\
\hline & Ordinary level certificate & 30 & 13.3 \\
\hline & Advanced level certificate & 14 & 6.2 \\
\hline & Professional certificate & 71 & 31.6 \\
\hline & Professional diploma & 65 & 28.9 \\
\hline & First degree & 21 & 9.3 \\
\hline & Postgraduate diploma & 11 & 4.9 \\
\hline & Master's degree & 9 & 4.0 \\
\hline \multirow{9}{*}{$\begin{array}{l}\text { Job specialty of } \\
\text { respondents }\end{array}$} & Administrator & 35 & 15.6 \\
\hline & Doctor/physician & 2 & 0.9 \\
\hline & Clinician & 19 & 8.5 \\
\hline & Laboratorian & 18 & 8.0 \\
\hline & Nursing/midwifery & 78 & 34.8 \\
\hline & Politician & 44 & 19.6 \\
\hline & Health Assistant & 6 & 2.7 \\
\hline & Environmental Health & 1 & 0.4 \\
\hline & Others & 21 & 9.4 \\
\hline \multirow{7}{*}{$\begin{array}{l}\text { Maximum number } \\
\text { of years worked in } \\
\text { job the specialty } \\
\text { area }\end{array}$} & $0-4$ years & 80 & 35.7 \\
\hline & 5-9 years & 48 & 21.4 \\
\hline & $10-14$ years & 32 & 14.3 \\
\hline & $15-19$ years & 26 & 11.6 \\
\hline & 20-24 years & 24 & 10.7 \\
\hline & $25-29$ years & 11 & 4.9 \\
\hline & Over 29 years & 3 & 1.3 \\
\hline
\end{tabular}

Source: Primary Data.

Just over 60 per cent of the respondents attained either a professional certificate $(31.6 \%)$ or a diploma $(28.9 \%)$. A significant proportion $(21.3 \%)$ had less than a professional certificate, majority of who are likely to be politicians, who constituted 19.6 percent of the respondents. The nursing/midwifery profession dominated with 34.8 percent. The minimum required credential for recruiting nurses and midwifes in healthcare service is a professional certificate. Physicians constituted only less than one per cent of the respondents.

With respect to the key informants, all were male, which suggests that men dominated higher occupational hierarchies than their female counterparts. One of the interviewees was a political scientist. Others were senior nursing officer (1), principal medical officers (2), senior clinical officer (1), senior medical officers (2), and medical officer (1). All the nine informants attained at least a post graduate diploma, and accumulated specialty experience ranging from 7-15 years. 
DOI: $10.21522 /$ TIJPH.2013.06.03.Art007

ISSN: $2520-3134$

\section{Barriers to EBMgt healthcare decision-making Limited research participation}

At least 53.8 per cent of all the survey respondents reported that they had never participated in any healthcare research undertaken in their work place in the last two calendar years. Only about 11.6 per cent said that they participated always in the research. This finding was in agreement with opinion of the key informants, who also decried of inadequate participation.

No, not adequate at all, I would like to participate better than this, not simply as a respondent or an entry point for researchers (HM1); our participation in most cases has been in providing authorization to researchers, we are hardly engaged in the initial design, identifying the problem or in reviewing protocols $(\mathrm{ADM})$. In most cases, research is designed from the top with no or little input of end users, most which come as donor driven projects that may not match real pressing needs (HM2), we only get to know about the research at implementation stage, but if you come and identify a problem in my house without involving me, how would you expect me to own it? (HM1), and self-initiated research are very few in my workplace, at least I have not come across one, research always appears imposed upon us (HM4).

\section{Limited research knowledge and skills}

At least some 70.1 per cent of all survey respondents had not attended any research literacy training in their workplace in the last two calendar years. Even those who said that they attended a training, made reference to the research methodology module attended from school, research induction and review or dissemination workshops. This was a same finding when it came to the key informants. It is therefore tempting to elucidate that none of the respondents had attended research literacy training in their work place in the last two calendar years.

When asked specific questions on knowledge and skills with respect to the six stages of a typical research knowledge translation and utilization process described by Barends et al. (2014), indeed a total of 65.6 per cent either knew nothing and lacked practical experience, or knew some theory but lacked experience. Only 4.8 per cent on average said that they knew quite a lot and needed no assistance. The extent of knowledge and skills gap was a major concern to some of the key as well informants. For instance, one had this to say:

Indeed, I sometimes have reservations on findings of some of the researches undertaken around us; as per now, our human resource capacity is very low to get good research outcomes from our laboratories; people sometime shun wrong out results, for instance, in a number of cases, diseases like malaria appear to be misdiagnosed; actually, a research was done recently to show that we have a lot of errors from our laboratories, making it quite difficult to rely on health researches like clinical trials undertaken in our local laboratories, more-over the clinicians too in many cases order for wrong investigations (ADM).

In these statements, the informant implies that local researchers have limited capacity and are therefore less competent, and suggests that research undertaken exclusively by local researchers are less likely to be utilized than those done in collaboration with international expatriates. Even so, there were mixed concerns regarding hired consultants. Some informants expressed concern that consultants hardly appeared on ground, but instead rehired less qualified local personnel with little mentoring, guidance and supervision.

\section{Limited access to internet facilities}

Only 9.3 per cent of all the survey respondents said that they accessed online journals on a frequent basis in the last two calendar years. About 28.0 per cent never accessed online journals, while 68.0 per cent said they rarely accessed online journals due to lack of internet facilities in their work place. Sure, it was a general consensus among the informants that internet facilities were largely lacking because most health facilities had to electricity and computers.

\section{Research attitude, perceptions and belief related barriers}

Although the survey reveals that the respondents generally had a more positive attitude towards research, a significant proportion (34.7\%) was inconsistent when it asked about their willingness to use research findings even if it contradicts knowledge learned from school years ago. They either disagreed or were not sure. More-over, an informant warned that research was still perceived as an alien thing, and an end-point in itself, which does not go beyond dissemination! 


\section{Leadership barriers}

At least some 22.6 per cent of all the survey respondents had a reservation that their leaders were supportive and compelled positive change and excellence in healthcare management. In fact, one key informant expressed a strong concerned that some of their leaders were not supportive enough due to conflict of interests, as reflected in the following statement.

Unfortunately, a good section of our leaders have been taken up by egoism and individualism, they look at what would benefit them first; what is my take in this; what will I benefit from it; yet if leadership doesn't take it up, certainly the followers won't take it too (HM2).

\section{Cultural barriers}

Up to 35.7 per cent of all the survey respondents expressed fear that the way things are done, the norms and values in their work place were not supportive to work and good change in healthcare management. Likewise, the key informants stressed the potential influence of culture on research evidence utilization. They augured that cultural factors like traditions, norms, values, practices and the way that things have been done over time, if not taken well care-off, can negatively affect utilization of a well-intended research. It was also noted that most managers tend to have a poor reading culture that makes them not aggressively seek for written information. Below are verbatim quotations from some of the interviewed key informants.

In number of instances, written information, more-so if bulky, are never read, they are filed up to dust, this is even worse for managers and leaders with busy schedules (HM7); besides, it's a common tradition that cure is only by injection, such beliefs tend to impede preventive approaches that may have been recommended by research (HM6).

\section{Understaffing and over work load}

Approximately 72.4 per cent of the survey respondents disagreed that level of staffing in their work place was enough staff to get necessary work done. Even among the informants, it appears a common consensus that understaffing and workload was a likely barrier, though one informant differed, and instead implicated attitude and cultural degeneration that affect work commitment.

I agree that the level of staffing may not be adequate in most health facilities, but that is not the root problem, the problem is attitude; increasingly, people are getting to the health profession not from a conviction to serve but rather as an employment; the health sector is currently suffering from cultural degeneration, our staff don't just develop the required commitment, it is not uncommon to find a staff in the quarters yet patients are waiting to be attended to (ADM).

\section{Lack of time}

A total of 60.5 per cent $(\mathrm{N}=221)$ of the survey respondents expressed concern that their work schedules could not allow them time to search and get acquainted with what happens around the world. Similarly, informants underscored the importance of time in influencing research use. They made arguments that their work environment would not allow time to do something else or differently. However, they indicated also that sometimes stakeholders fail to appreciate the benefits of research because many researchers hardly give enough time to explaining the benefits and purpose of research. These views are depicted in some of the verbatim quotations below.

It requires time because the process is technically demanding (HM6); you must have time to retrieve, review and evaluate available evidence before putting it to use, in most cases we lack this time (HM2); likewise, researchers must have the time to explain the purpose and benefits of the research (HM2 \&3), but unfortunately, many researchers come in a rush, they have no time, they have no patience, somebody comes in 10 minutes wanting you to complete a questionnaire, even when I have not understood or appreciated the research (HM2), they will tell you fill here, fill there (HM3).

\section{Limited formal interactions}

Formal interaction may take the form of formal departmental or unit meetings, circulars, workshops, seminars and conferences. Lack of interactions inhibits information sharing. At least 62.0 per cent $(\mathrm{N}=221)$ of our survey respondents indicated that they never or rarely participated in formal departmental or unit meetings in their work place in last typical month. 


\section{Perceived quality}

At least 33.3 per cent of all the survey participants indicated that research had provided evidence not of sufficient quality to make best healthcare management decisions in their work place. In this survey perceived quality had a significant positive influence $(\mathrm{P}=0.040,95 \% \mathrm{CI})$ on research utilization, with odds ratio high as 4.093 , which implies that a research that is perceived to be of sufficient quality was four times more likely to be utilized. However, it follows that there were diverse perceptions of what amounted to quality research, as reflected in the following verbatim quotations. This diversity represents the level of challenge it to satisfy expectations of the participants, and the extent to which quality is likely to influence research utilization.

Action oriented research done within the locality of the area (HM5); addresses the real needs of the target end users (HM6); a research that provides implementable recommendations (HM3); provides beneficial solutions to existing problems, and harnesses the existing local practices and policies (HM2); done timely and results disseminated when still relevant (HM6); the findings are able to be used (ADM); also caused some desirable change (HM7); a research that generates information from correct informed sources, because people don't always lie, they will always tell the truth especially if it targets a problem that affects their lives (HM3); a research that involves correct stakeholders right from its conception, inception, throughout its implementation, dissemination to consumption (ADM); collaborative research (HM3); done together with the local people (HM2); scientific research (HM6); whose write-up uses appropriate audience tailored language (HM8); adequately funded not to compromise outputs (ADM).

In short, research quality is a multifactorial concept that manifests in various forms depending on the end user. Key aspects of research quality include; relevance, strength (credibility \& reliability), practicability, objectivity, accessibility, as well as dissemination methods and timing. In this survey, relevance of evidence of had a positive significant influence $(\mathrm{P}=0.019,95 \% \mathrm{CI})$, with corresponding odds ratio of 3.940, which implies that a research considered as relevant was about four times more likely to be utilized. Likewise, practicability of evidence had similar odds ratio, and positive significant influence on research utilization $(\mathrm{P}=0.009,95 \% \mathrm{CI})$.

\section{Cost related barriers}

It emerged during the key informant interviews that cost of accessing and retrieving research evidence was an important uptake barrier. Lack of internet at work place meant that healthcare authorities had to use personal funds to purchase internet bundles or visit internet café. With exception of some senior managers and administrators no airtime allowances.

\section{Poor record keeping}

Even when researchers attempt to provide hard copy findings, sometime the document is delivered, but no one knows it's about, or we keep it in a way that it can't be accessed with ease if required for making reference in decision-making; in most cases the hard copies are lost or personalized because we lack proper official record keeping (HM5).

\section{Poor dissemination methods and timing}

The survey observed positive, but insignificant relationships between research utilization and dissemination methods $(\mathrm{P}=0.218,95 \% \mathrm{CI})$ and timing $(\mathrm{P}=0.290,95 \% \mathrm{CI})$, with corresponding odds ratios of 1.984 and 1.832 respectively. Indeed, the key informants expressed concerns over improper methods and timing of dissemination. For instance, one informant exclaimed that:

To me, there is a problem in dissemination, they don't put in account who is going to use the findings, if a research targets local government and you put it on internet, no one will certainly access it! (HM7); sometime the language used is too technical to be easily understood by a layman (HM5); then others disseminate the findings when they are no longer useful to solving the problems for which it was intended (HM4).

\section{Researchers' competence, authority and trust related constraints}

Authority of researchers $(\mathrm{P}=0.019,95 \% \mathrm{CI})$ and trust in researchers $(\mathrm{P}=0.006)$ both had significant positive influence on research utilization. The corresponding odds ratio with respect to trust was 3.147, while that for authority was 1.885 . Competence of researchers had a positive insignificant relationship $(\mathrm{P}=0.885)$ with research utilization in management decisions, and a corresponding odds ratio of 1.915. More-over, the key informants stressed researchers should be 
undertaken by highly competent personnel so that the findings reflect a true situation. Some informants expressed fear that research undertaken exclusively by local researchers are less likely to be utilized than those done in collaboration with international expatriates. There were also mixed concerns over hired consultants, that most of them do not get to field to undertake the research, but instead hire research assistance who in most cases lack relevant skills.

Trust builds confidence and inspires people to use research evidence; you cannot make use of information don't trust the source (HM7); this certainly has to do with competence but if I do not trust the results, how would you expect me to spearhead making use of it as a leader? (ADM)

\section{Limited communication}

This survey reveals a positive insignificant $(\mathrm{P}=0.122,95 \% \mathrm{CI})$ influence of communication between researchers and end users, with a corresponding odds ratio of 1.871 , implying that where communication is open and free, findings are about twice more likely to be utilized. The key informants add that when researchers communicate with end users in a freely open manner, trust builds up, which results in increased research utilization.

\section{Low policy enforcement}

Though it was appreciated that fine policies were made, there were obvious gaps in enforcement and evaluation, yet some of these research are intended to address gaps identified in policies. Certainly, it is logical that if such a policy is not implemented, the findings from such a research would not be taken up. The conflict between policy and politics was eminently spelt out, whereby politics tend to antagonize recommendations and implementation of a good policy. For instance, the policy says do this, but the politician will say no, simply because it affects votes.

\section{Strategies to increase EBMgt healthcare decision-making}

Following the interviews with key informants, a number of opportunities were eminent for increasing research evidence uptake. We have categorized these measures under five broad areas; engagement and participation, dissemination, capacity building, leadership and governance, and finally knowledge marketing and visibility.

\section{Adequate stakeholders' engagement and participation}

It was a common consensus among the key informants that right stakeholders must be engaged right from the beginning of the research. Stakeholders should be engaged in identifying and analyzing the research problem so that the findings address the real needs of the local people. In short, the bottom-up design is most appropriate. For instance, some informants emphasized that:

Research should be based on a real need and provide feasible solutions to existing problems (HM2), but this is only possible if right stakeholders are rightly involved right from the beginning (HM3); implementation is about politicians, so long as we don't involve them, it may not succeed however good the evidence is, it must ensure that interests are met (HM8), and it should be a round holistic engagement, right from conception, inception, implementation, dissemination and consumption (ADM).

However, some informants were cautions that the stakeholders to be involved have to be the right ones, which calls for a need for a careful stakeholders analysis while designing research. With inference to "consumption", the informant implies that the mandate of researchers does not stop at just dissemination, but researcher should take possible measures to ensuring the findings are actually put to use. In view of these arguments, collaborative research that adopts bottom-up approaches were recommended as most effective approach because it harnesses local ownership, builds local capacity, attracts funding, and ensures sustainability of the interventions, as reflected in this verbatim quotation.

There is a need to support local entities to undertake research so as to guarantee sustainability; the best approach would be to make research collaborative owing to the capacity and funding limitations of such local entities (HM2).

\section{Contextualized dissemination}

Secondly, the informants placed emphasis on contextualization of dissemination so as to enhance accessibility. Below is what some of them had to say:

The most ideal situation would be to disseminate at various levels so that the information package can be tailored to the varying needs of the target audiences (HM4); besides person to person 
dissemination would be most appropriate for key target stakeholders like leaders (ADM); however, if resources are limiting, group dissemination, perhaps at district level would be considered (HM1).

In such group dissemination, a careful selection of key stakeholders was recommended so that the audience reflects a complete representation of target users. Involvement of the media was over stressed as the fourth arm of the state, arguing that they play a pivotal role in transferring the information to a wider audience. Others were opinion leaders that often acted as agents of positive change. Further, it was argued that local staff should be given the opportunity to present the work by themselves. For instance, one informant attests that:

People will tend to own the findings better if they listen to it from their own personnel, and discuss it with them, sometime, it may be necessary emphasize in local language for better understanding (HM3).

And, whereas consultants and experts were regarded to be more competent with more authority, the informants advise that they play more of a guiding and mentoring role. More still, they advise that during dissemination meetings, summaries on benefits of the research should be presented and discussed, and any arising issues must be resolved at this point. These are reflected in some of the verbatim statements cited below.

The benefits of the research must be prominently articulated (ADM); summarize findings in a way that best suit the audience (HM6); the audience must be given ample time to critically discuss the findings (HM2); it is important that concerns are addressed at this point, a dialogue is essential here to arrive at a win-win situation, otherwise they will disown the findings (HM5).

Indeed, these statements depict how crucial dissemination can influence research uptake, and that the platform should not only be used to share findings but resolve arising disputes and differing opinions so that the findings can be owned by all participants. Some informants continued to warn that:

One common mistake in dissemination workshops is not to come up with action points, or an action plan, way ford or resolutions, which makes dissemination as an end point to research (ADM); the audience must be tasked, they must make clear their commitments so that they feel accountable (HM2); besides, there should be hard copies summarizing the key findings and recommendations (HM6).

Considering local government setting, hard copy dissemination was generally preferred to electronic because most health facilities lacked computers and internet. As a matter of best practice, the hard copies originate from district top leaders or bear evidence of formal ownership. Finally, timing of dissemination was another point of emphasis, that the findings must be disseminated when still relevant to end users.

\section{Local capacity building}

All researches must have the component of capacity building, if it is to be owned and sustained (ADM, HM2, HM5); train a core team of local staff that can undertake research on their own so that they can train others, there is nothing more critical than skills themselves, skills in itself is a strong motivation to move forward and look for alternative way of doing things better, but you cannot get to know that you actually do not know, unless you have been shown that you do not know; our staff need to be challenged in terms of knowledge (ADM).

According to the informants, when health workers acquire required skills, they will be motivated to look for new knowledge on how to do things differently, thereby promoting the search for new evidence, which is one of the steps in utilization. While the constraint of cost was eminent, some of informants argued that cost is only a secondary inhibitor, as depicted in statements below.

The issue of financial constraints will certainly be overcome, so long as people have the right skills (ADM); for instance, they can integrate research activities in other on-going funded areas (HM5); the starting point is that people must demonstrate appropriate skills in order to convince decision makers of a need for funding, they should be in position to demonstrate why funds should be put in research (ADM).

Certainly, the informants suggest that appropriate skills are primary for the workforce to integrate activities and advocate for increased budgetary allocations to research. Some of the avenues that were identified as potentially feasible for transferring knowledge and skills to local healthcare personnel include engaging them in the research activities, in a manner that provides learning, on job mentoring and training, and short research literacy tailored training. Besides, it was recommended that trainings should be coupled with appropriate infrastructural and equipment needs as reflected in the below. 
These must go hand in hand, but unfortunately, most funders tend to put more emphasis on equipment or infrastructure and ignore the HR component; in any case, who would make use of the facilities if the project came to an end; we have seen equipment abandoned in hospitals because local staff cannot operate them (ADM).

Sure, the ADM was concerned of imbalances in investments on research equipment and infrastructure, against human resource capacity development, that it threatens sustainability. He supported this point by giving example of hospital equipment, which have not been put to proper use due to lack of human resource capacity. More-over, it follows that authority was interpreted in terms of competence, which provides the ability to command self-respect from the end users. They closely linked trust with competence and authority, with some arguing that:

Trust builds confidence and inspires people to use research evidence; you cannot make use of information don't trust the source (HM7); this certainly has to do with competence but if I do not trust the results, how would you expect me to spearhead making use of it as a leader? (ADM)

\section{Knowledge marketing, awareness and visibility}

Just like any business venture, the need to brand and market research evidences is essential. The target audience needs to know of the outputs, outcomes and benefits from a research. Some of the measures identified as most appropriate for achieving this include; continuous awareness, education and dialogues so as to avert negative cultures, attitude, perceptions and beliefs about research, and make users appreciate the benefits of integrating best scientific evidence in healthcare management decision-making and practice.

The informants argued that linkage between researchers and end users was stronger in researches that adopted a bottom-up design approach. According to the informants, it is important to create a mass of change agents that will assist with marketing the research and its findings so that users become familiar with the benefits of research, and make research part of their usual activities. With respect to communication, below are what some of the informants had to recommend in order to increase visibility of research:

Depending on the purpose, researchers need to open-up to various communication channels that are currently available, exploit the boom in information technology, including emails, SMS, social media, print media, radios and televisions among others (HM5), there is need to increase visibility of research (HM7).

\section{Local leadership and governance}

As one key informant outs it, "implementation is all about politicians and leadership". It was emphasized that good leaders should provide the required inspiration, motivation and stewardship that compel positive change in healthcare management decision-making in their workplace. Consequently, efforts to promote research evidence utilization must first be embraced by local leaders, right from the district to lower levels, more-so that they determine resource allocations, guide policy directions, and play a key role in mobilizing people in any healthcare system. But, the question of getting good leaders remains a big challenge more-so in corrupt systems, how do we have pro-people leaders with no selfish interests, individualism and egoism? Nonetheless, the informants stress the keep leaders continuously reminded, accountable through ways like performance scoring, public dialogues and. In fact, one informant stressed that no leader would like a low score! There must be a clear distinction between policy and politics.

\section{Discussion}

In view of the merits attributed to mixed methods that is making such approaches gain increased prominence and recognition by most researchers (Zohrabi, 2013), this study combined both qualitative and quantitative methods and instruments to simultaneously collect empirical field data so as to enhance validity and reliability of findings by permitting triangulation, verification, enrichment, confirmation, complementarity, reinforcement and thorough explanation of findings through in-depth interviews (Caracelli et al., 1997; Kelle, 2008, Bamberger, 2012 \& Zohrabi, 2013) with selected key informants. Through descriptive statistics, this study was able to establish proportions of respondents in agreement or disagreement with the importance of the identified barriers and promoters of research uptake, and in some cases established the extent and significance of the factors through modeling.

But why was this study important? While one would perhaps make the argument that literature on barriers to and promoters of evidence are in abundance, as in fact mentioned by one of the key informant in this study that they are tired of studies that simply look at factors, it is important to be 
informed that these factors differ in contexts. The differences are not only between developed and developing worlds, but also within the developing countries themselves, as well as between sectors. Besides, we note that only 5.5\% of all studies reviewed by (Oliver et al., 2014) provided strategies for increasing uptake, while majority (59.3\%) focused on simply identifying barriers to and/or facilitators of research evidence use.

We identified both barriers to, provided strategies for increasing research evidence use, and think that the results may to a certain extent be generalizable to the local government healthcare setting of Uganda, since we used a mixed approach. Nonetheless, readers must bear in mind that barriers in one context may actually be promoters in another context. This is well evident in the recent systematic review by Oliver et al. (2014). For instance, they observed that while 49 studies affirm collaboration as a promoter, eight studies reported it as a barrier. Likewise, eight studies reported contact with researchers as a barrier, yet 31 studies noted it as a promoter. In fact, all the factors they identified manifested as a barrier, but also as a promoter in another context! This justifies the need to replicate these studies in different contexts.

Another strength in the present study is that it analyzed the utilization of research at healthcare decision-making level. We know that decision-making is central in an any healthcare systems, whether at practice or policy level. More-over, the study targeted healthcare authorities a cross all levels of the local government decision-making hierarchy.

The findings from this study are not much different from those reported by earlier studies elsewhere. For instance, a recent systematic review (Rengerink et al., 2013) highlighted a number of barriers to utilization of research evidence. They highlighted lack of time to search and read available evidence, lack of resources or facilities, and lack of skills for evidence based practice. This study identified the lacking resources and facilities to include internet, computers, electricity and records keeping facilities, including libraries. Sure, in many instances, the informants decried of lack of internet connectivity, computers and electricity in most healthcare facilities, yet the personnel were not or if so were poorly facilitated with allowances. The significant role of access to internet and libraries is supported further in literature by Squire et al. (2013). Likewise, Albert et al. (2007) emphasized access to information as being an important barrier to research uptake in Mali healthcare system.

Also, Squire et al. (2013) describe the influence of organizational leadership and culture on research evidence use, in that positive outcomes are generally associated with leadership and cultures that are more people oriented, less controlling and more relationship based. In addition, they highlight the importance of information sharing (social interaction) and formal interactions such as through departmental meetings. While Albert et al. (2007) observed authority and accountability as two newly emerging issues in literature, the dual were equally emphasized by informants in this study. Certainly, leadership, politics and governance go hand in hand.

The significant role of quality of research and its evidence is detailed in literature. For instance, Oliver et al. (2014), Albert et al. (2007) as well as Nabyonga et al (2012) reported at least one or more of the following attributes related to perceived quality; the nature and strength of evidence, relevance, credibility, reliability, feasibility, timeliness, and objectivity of evidence, authority and competence of those who undertake research, as well as appropriateness of research design, implementation and dissemination strategies. This study substantiates the diversity with which research quality and evidence perceptions manifest in healthcare systems. For instance, in addition to the above attributes, issues related to locality within which research is undertaken, sources of data (generating information from correct informed sources), the need for adequate funding of research activities, and a strong emphasis on collaboration and all round stakeholders' participation emerged out eminently during the interviews. This diversity reflects the extent of challenge researchers are faced in meeting expectations of potential users of evidence.

Cost as a barrier to research evidence use was underscored too, which adds voice to the 25 studies Oliver et al. (2014), reported as having identified cost as a barrier to research use. It is however not clear on the circumstances in which at least 11 studies they reviewed reported cost as a promoter of research uptake. However, this may be much surprising because most people in the rich class tend to belief that anything free is not of quality, and the more expensive an item is, the more quality in it. This could apply as well to free access journals visa vie those that require money to access.

The healthcare authorities interviewed in this study expressed concern that searching, accessing and reviewing journals is highly time consuming, yet they are already workload burdened due to understaffing in most healthcare facilities. In this case, systematic reviews may appear a feasible alternative, even though none of the participants (both informants and respondents) mentioned 
systematic reviews as source of information they used to make healthcare management decisions in their workplace, which likely due to lack of internet, cost, and perhaps the fact that majority of them may not be aware of systematic reviews. Systematic reviews are increasingly more recognized as offering high quality evidence for clinical practice (Mulrow, 1995), including the fact that they lead to a more efficient use of time (Lavis et al., 2005).

With respect to increasing research utilization, the strategies recommended in the present study have a strong backing in literature, except that we have contextualized and detailed them. For instance, stakeholders' engagement and participation is strongly supported by Sauerborn, Nitayarumphong and Gerhardus (1999). Nonetheless, while they recommend in their study that the only way to guarantee ownership is to make leaders (policy makers) commission a research, informants in the present study demanded their participation in research will only be adequate if they are engaged at all stages of the research process, right from identification and analysis of the problem, designing and reviewing the study, implementation, dissemination and feedback.

In fact, one informant described it as "a round holistic engagement, right from conception, inception, implementation, dissemination and consumption, (ADM)". The bottom-up approach was strongly recommended in favor of top-bottom approach, and while collaborative research was over supported, we did not establish if the local stakeholders were willing to co-fund research activities in their workplace. The Overseas Development Institute (ODI, 2009) in their planning toolkit, asserts that identifying which stakeholders to engage, and how to engage them in any given research calls for a careful analysis to establish interests, influence and power, as well as potential impact of the research to their livelihoods and practice. But generally, the role of the private sector like the must not be under looked (ODI, 2009).

However, there was a common view was that if staff had the necessary skills and competencies, they would be in position to lobby and convince their leaders to allocate funds for research. It was argued that competent would be in position to integrate research into other funded activities. Sure, Lansang and Rodolfo (2004) emphasized the need for local capacity building as a strategy for increasing research uptake. We think that the informants' call for a strategically balanced in human, equipment, and infrastructural investments was sound towards ensuring sustainability of the interventions as some of them lamented that most equipment become redundant in hospitals because the project did not invest in building capacity of local personnel to operate the machines. This view is well supported by Sauerborn et al. (1999) who stressed the need to make the use of health systems research sustainable by creating an appropriate institutional framework. In fact, the key informants recommend that all research must have a component of capacity building, while Wallen et al. (2010) backs on-job training, mentoring and coaching as being cost-effective.

The third strategy that we recommend relates to contextualized dissemination of findings, ensuring that the communication process, products and timing are appropriate to local needs of the stakeholders, and that all relevant stakeholders are represented. This recommendation is strongly supported by Sauerborn et al. (1999, p832), who phrased it as "adapting the communication process and products to stakeholders' needs". The language used must match the levels of target audience. This implies that researchers should consider several avenues of disseminating evidence. Short simple summarizes were strongly recommended as best, more-so for busy leaders and managers. Similarly, making local personnel present findings during conferences, and where possible use local languages, was not only viewed as way of building capacity but also harnessing ownership. Besides, there were observations that subordinates were more likely to utilize findings have been endorsed by their superiors. For this reason, we opted to include the District Health Officer (DHO) and Assistant Chief Administrator for Health (ACAH) in this publication, also in recognition for their contribution to this study as key informants, approving the study, and mobilizing participants.

The forth strategy highlights the importance of knowledge marketing and visibility, creating agents of positive change. This is because even that most healthcare authorities recognize the role of research in shaping healthcare management decisions, the benefits of some of the researches were not clear, according to the key informants. Besides, "research is still considered as an lien thing, which is an end in itself and does not go beyond dissemination", one of the key informants asserts. Consequently, they recommend that need to market and increase visibility of research and its products must draw equal important as any other business.

Fortunately, the concept of organizational knowledge marketing or brokering is not new (Hsu \& Lim, 2014). The brokers are simply people or organizations that move knowledge around and create connections between researchers and their various audiences (Meyer, 2010), and are recognized in literature as vital bridges or intermediaries (Verona, Prandelli \& Sawhney, 2006; Lavis, 2006; Lyons 
et al., 2006; Lomas,2007) that link producers and users of research evidence so as to facilitate collaborations and solve problems. And, according to Robeson et al. (2008) and Dobbins et al. (2009), they facilitate the appropriate use of the best available research evidence in decision making processes, and enhance individual and organizational capacity to participate effectively in evidenceinformed decision making. In the context of the present study, social networks such as the booming social media was identified as a potential voluntary inexpensive knowledge brokering channel. This is in addition to local radios and televisions. Besides, almost all public servants read newspapers for one reason or another, such as passing time.

Finally, strategy number five was about promoting best practices in organizational leadership and governance, based on the concern that implementation is all about leaders because they inspire, motivate, guide, mobilize, formulate and direct policies, and decide resource allocations. The importance of accountability was reported by Albert et al. (2007) based on a qualitative study in Mali's healthcare system. Among the methods the key informants in this study recommend as appropriate for holding public leaders accountable include; performance scoring, public dialogues or commonly known as the "baraza". Indeed, Kyohairwe (2014) underscored the importance of barazas in promoting local democracy and public accountability in Uganda's local government system of administration. Fortunately, in 2009, Tumushabe et al. (2010) launched a 10 years initiative to performance of Uganda's Local Government Councils. Perhaps this should be scaled up to include all districts in Uganda. In short, we equally strongly agree with these observations, and wish to reinstate that leaders provide strategic direction in an organization.

In conclusion, we wish to reinstate that barriers and promoters of research evidence use vary depending on local context, in some instances a barrier in one context is a promoter in another context. On this basis, we recommend that studies on factors for research utilization be replicated in various contexts. We further recommend that such studies should focus on identifying feasible local contextualized strategies to increase research evidence use so as to optimize the benefits of research.

\section{Ethical declaration}

We a test that this research was undertaken according required ethical principles and guidelines, that the participants were treated in accordance with the ethical principles of research as guided by UMI's Research Ethics Committee (REC), and the Uganda National Council for Science and Technology (UNCST), as per protocol review and approval letters referenced G/35 and SS 3826.

\section{Acknowledgements}

We wish to recognize the following individuals for helping with administering the questionnaires; Mr. Titus Apangu, Ms. Janet Abaru, Mr. Lawrence Owacuwun, Mr. Lawrence Ojosia, Mr. Felix Okoth and Ms. Harriet Apio. We must emphasis that the quality and integrity of the data was largely due to your strict adherence to required ethical procedures. In the same spirit, we acknowledge the research participants for voluntarily accepting to give us data. Special thanks to all the key informants for their lengthy time the Medical In-charges for Kuluva Hospital, Oli, Rhino Camp and Omugo Health centers IV.

\section{References}

[1]. Albert, M. A., Fretheim, A., \& Maïga, D. (2007). Factors influencing the utilization of research findings by health policy-makers in a developing country: the selection of Mali's essential medicines. Health Research Policy and Systems, 5 (1), 2.

[2]. Amanda, J. F., Dennis, F., Tara, J. S., Joseph, G.D., LeAnn, E.B., Shawn, C. O.\& Bibhuti, K.S. (2009). The Challenges of Implementing Evidence Based Practice: Ethical Considerations in Practice, Education, Policy, and Research. Social Work and Society International Online Journal, 7 (2).

[3]. Amin, M. F. (2005). Social Science Research: Conceptions, methodology and Analysis. Makerere University, Kampala.

[4]. Bamberger, M (2012). Introduction to mixed methods in impact evaluation. InterAction. Retrieved from http://www.interaction.org/sites/default/files/Mixed\%20Methods\%20in\%20Impact\%20Evaluation\%20\%28Engl ish\%29.pdf.

[5]. Barends, E., Rousseau, D. M., \& Briner, R. B. (2014). Evidence Based Management: The Basic principles. Center for Evidence-Based Management.

[6]. Caracelli, V., Greene,J., \& Jennifer, C. (1997). "Crafting mixed-option evaluation design." In J. C. Greene and V. J. Caracelli (eds.), Advances in mixed-option evaluation: The challenges and benefits of integrating 
diverse paradigms. New Directions for Program Evaluation, No. 74. San Francisco, CA: Jossey-Bass, pp. 1932.460

[7]. Dobbins,M., Paula, R., Donna, C., Steve, H., Roy, C., Linda O'Mara., Kara, D., \& Shawna, M. (2009). "A description of a knowledge broker role implemented as part of a randomized controlled trial evaluating three knowledge translation strategies". Implementation science, 4, 23.

[8]. Fraser, I. \& Clancy, C. M. (2007). Forward: Importance of adopting evidence-based management in healthcare. https://www.ache.org/pubs/pdf_preface/Kovner_Foreword.pdf.

[9]. Gwenyth R. Wallen, G. R., Sandra A. Mitchell, S.A., Melnyk, B., Fineout-Overholt, E., Miller-Davis, C., Yates, J. \& Clare Hastings, C. (2010). Implementing evidence-based practice: effectiveness of a structured multifaceted mentorship programme. J Adv Nurs, 66(12): 2761-2771. doi: 10.1111/j.1365-2648.2010.05442.x

[10]. Hsu, D.H., \& Lim, K. (2014). Knowledge Brokering and Organizational Innovation: Founder Imprinting Effects. OrganizationScience, 25 (4), 1134-1153.

[11]. JEBMH (2014). Journal of evidence based medicine (JEBMH). Retrieved from: http://www.jebmh.com/bibliographic.php.

[12]. Kelle, U. (2008). Combining qualitative and quantitative methods in research practice: purposes and advantages. Qualitative Research in Psychology, 3 (4), 293-311.

[13]. Kline, P. (2000). Handbook of Psychological Testing (2nd Ed). London: Routledge.

[14]. Kothari, C. R. (2004). Research Methodology: Methods and Techniques (2nd ed.). New Age International Publishers.

[15]. Kyohairwe, S. (2014). Local democracy and public accountability in Uganda: The need for organizational learning. Commonwealth Journal of Local Governance, Issue 15, Retrieved from: http://epress.lib.uts.edu.au/ojs/index.php/cjlg.

[16]. Lansang, M. A., \& Rodolfo, D. (2004). Building capacity in health research in the developing world. WHO, $82(10)$.

[17]. Lavis, J. N (2006). "Research, public policymaking, and knowledge-translation processes: Canadian efforts to build bridges". The Journal of continuing education in the health professions, 26 (1), 37-45.

[18]. Lavis, J., Davies, H., Oxman, A., Denis, J. L., Golden-Biddle, K., \& Ferlie, E. (2005). Towards systematic reviews that inform health care management and policy-making. J Health Serv Res Policy 10 (1), $35-$ 48.

[19]. Lomas, J. (2007). "The in-between world of knowledge brokering". BMJ (Clinical research ed.) 334 (7585), 129-132.

[20]. Lyons, R., Warner, G., Langille, L., \& Phillips, S.J. (2006). "Piloting knowledge brokers to promote integrated stroke vare in Atlantic Canada.". Canadian Institutes of Health Research (CIHR) Institute for Population and Public Health, editor. Moving population and public health knowledge into action: A casebook of knowledge translation stories. Ottawa, ON: Canadian Institutes of Health Research (CIHR) Institute for Population and Public Health.

[21]. Meyer, M. (2010). The Rise of the Knowledge Broker Science Communication, 32 (1), 118-127

[22]. Melnyk, B. M., Fineout-Overholt, E., Gallagher-Ford, L., \& Kaplan, L. (2012). The state of evidencebased practice in US nurses: Critical implications for nurse leaders and educators. The Journal of Nursing Administration, 42(9), 410-417.

[23]. Mulrow, C. D (1995). Rationale for systematic reviews. Systematic Reviews. Edited by: Chalmers I, Altman DG. 1995, London: BMJ Publishing Group, 1-8.

[24]. Nabyonga, J., Bataringaya-Wavamunno, J., Bakeera, S. K., \& Criel, B. (2012). Do guidelines influence the implementation of health programs? - Uganda's experience. BioMed Central, 7(98), 1-16.

[25]. ODI (2009). Overseas development Institute (ODI) planning tools: Stakeholder Analysis: Toolkits. Retrieved from: http://www.odi.org/publications/5257-stakeholder-analysis

[26]. Oliver, K., Innvar, S., Lorenc, T., Woodman, J \& Thomas, J. (2014). A systematic review of barriers to and facilitators of the use of evidence by policymakers. BMC Health Services Research, 14, 2, 1-12.

[27]. Paula, R., Dobbins, M., \& Decorby, K. (2008). "Life as a knowledge broker in public health". Journal of the Canadian Health Libraries Association (JCHLA/JABSC), 29, 78-82.

[28]. Pfeffer, J., \& Sulton. I, R. (2006a). Decision-making: Evidenced-based Management. Harvard Business Review, 84 (1), 63-74. Retrieved from https://hbr.org/2006/01/evidence-based-management

[29]. Pfeffer, J. \& Sutton, R. (2006b). Hard Facts, Dangerous Half-Truths and Total Nonsense: Profiting from Evidence-Based Management. Boston, MA: Harvard Business School Press.

[30]. Sauerborn, R., Nitayarumphong, S., \& Gerhardus, A. (1999). Strategies to enhance the use of health systems research for health sector reform. Tropical Medicine and International Health. 4(12), 827-835. 
DOI: 10.21522/TIJPH.2013.06.03.Art007

ISSN: $2520-3134$

[31]. Sackett, D. L., Rosenberg, W. M. C., Gray, J. A. M., Haynes, R. B. \& Richardson, W. S. (1996). Evidence Based Medicine: What It Is and What It Isn't, in: British Medical Journal, 312, 71-72.

[32]. Squires, J. E., Hayduk, L., Hutchinson, A. M, et al. (2013). A protocol for advanced psycho-metric assessment surveys. Nurs Res Pract.

[33]. Squires, J. E., Estabrooks, C. A., Gustavsson, P., \& Wallin, L. (2011a). Individual determinants of research utilization by nurses: a systematic review update. Implementation Science, 6 (1),1.

[34]. Straus, S. E., Richardson,W. S., \& Glasziou, P et al. (2005). Evidence-based medicine, how to practice and teach EBM. Third edition. Edinburgh: Churchill Livingstone.

[35]. Tumushabe, G., Muyomba-Tamale, L., Ssemakula, E. \& Lukwago, D. (2010). Uganda Local Government Councils Score-Card Report 2008/09: A Comparative Summary of Findings, Conclusions and Recommendations. ACODE Policy Briefing Paper Series, Kampala.

[36]. Verona, G., Prandelli, E., \& Sawhney, M. (2006). "Innovation and virtual environments: Towards virtual knowledge brokers". Organization Studies, 27 (6), 765-788.

[37]. Zohrabi, M. (2013). Mixed Method Research: Instruments, Validity, Reliability and Reporting Findings. Theory and Practice in Language Studies, 3(2), 254-262. 